\title{
Desafios na construção de um modelo para análise comparada da organização de serviços de saúde
}

\section{Challenges for developing a comparative analytical model for health services}

Virginia Alonso Hortale 1

Eleonor Minho Conill 2

Manoela Pedroza 3

\footnotetext{
1 Departamento de Administração e Planejamento em Saúde da Escola Nacional de Saúde Pública, Fundação Oswaldo Cruz. Rua Leopoldo Bulhões 1480, 7 o andar, Rio de Janeiro, RJ 21041-210, Brasil. virginia@ensp.fiocruz.br

2 Departamento de Saúde Pública da Universidade Federal de Santa Catarina, Centro de Ciências da Saúde, Universidade Federal de Santa Catarina.

C. P. 476, Florianópolis, SC 88040-900, Brasil. eleonor@repensul.ufsc.br 3 Departamento de Administração e Planejamento em Saúde da Escola Nacional de Saúde Pública,

Fundação Oswaldo Cruz.

Rua Leopoldo Bulhões 1480, 7o andar, Rio de Janeiro, RJ 21041-210, Brasil.
}

Abstract This paper presents different models that describe health determinants, services, and systems. It highlights the important model devel oped by Contandriopoulos, proposing a separation between the health status and health care circuits. The article is intended as a contribution to the field of comparative analysis with a new theoretical, methodological, and evaluative approach. Problems were identified in the development of a new model. Decentralization and access are introduced as categories, suggesting that they may take part in such new model. Key words Health Services; Health System; Health Service Analysis

Resumo O artigo apresenta os diferentes model os propostos para descrever os determi nantes de saúde, os servi ços de saúde ou o sistema de saúde em geral. Aponta como promissor o modelo de senvolvido por Contandriopoulos, que propõe a separação entre circuito de estado de saúdee circui to de atenção à saúde. Coloca-se na perspectiva decontribuir, no campo da análise comparada, com um novo aporteteórico, metodológi co e avaliativo, explicitando a existência de problemas e encruzil hadas na tarefa de construir e operaci onalizar um novo modelo. São introduzi das as categorias descentral i zação e acesso, sugerindo-se que possam compô-lo. Palavras-chave Serviços de Saúde; Sistema de Saúde; Análise de Serviços de Saúde 


\section{Introdução}

Neste artigo, reproduzimos as discussões provenientes de estudo em desenvolvimento cujos objetivos são de construir e adequar modelo para análise comparada da organização de serviços de saúde. $O$ estudo atende a duas premissas básicas. A primeira diz respeito à intenção de incluir explicitamente a análise comparada no campo da avaliação, na medida em que consideramos que esta subentende um julgamento de valor da qualidade dos sistemas de saúde mediante um processo de análise sistemático e relativamente objetivo. A segunda refere-se à intenção de incorporar variáveis e dimensões históricas, político-administrativas e culturais ao modelo, para que possam traduzir não apenas a estrutura, mas também a dinâmica dos sistemas e dos serviços.

As três últimas décadas foram marcadas por uma discussão crescente quanto à crise eà necessidade de reformas nos sistemas de saúde. Os aspectos relacionados aos custos e à crise fiscal dos estados, ainda que enfocados sob ângulos diversos, foram, sem dúvida, aqueles aos quais se deu maior ênfase. Como pano de fundo, a globalização de uma tendência neoliberal nas políticas sociais. Nos países centrais, essa discussão se manifesta por al guns questionamentos da relação serviços-saúde, que, longe de estar equacionada, esforça-se por mostrar um quase colapso de meios e fins, no qual a existência de serviços não geraria, numa relação direta, a saúde.

Os principais questionamentos se baseiam em três constatações. Primeiro, tornar-se-ia cada vez mais patente a importância de fatores externos aos serviços na saúde de grupos populacionais (renda familiar, escolaridade, hábitos de vida, local de trabalho etc.). Segundo, uma análise mais rigorosa mostraria que as práticas médicas não estão tão estreitamente ligadas a um conhecimento científico como pode parecer. Em outras palavras, as expectativas sociais (e seus recursos) depositadas nos serviços de saúde estariam superdimensionadas, levando-se em conta o retorno real que elas podem dar. Por último, existiria uma grande distância entre a compreensão atual dos determinantes de saúde e as prioridades definidas para os serviços pelas políticas de saúde. Esta separação entre teoria e prática poderia ser decorrência da persistência na utilização de quadros de referência ou modelos incompletos ou obsoletos de conceituação dos determinantes de saúde para a concepção dessas políticas (Evans \& Stoddart, 1996).
Ainda que impregnadas por uma abordagem que tende a reduzir as análises do setor, desconhecendo, na maior parte das vezes, a subordinação da lógica dos serviços à ordem econômica mais geral, há uma concordância de parte de nossas discussões com este último ponto. Antes de compararmos os sistemas de saúde, é preciso rediscutir as bases e as categorias com que os serviços costumam ser avaliados.

O nosso objeto de estudo situa-se no plano da organização dos serviços. Propomos comparar as diversas realidades organizacionais, tendo em vista os problemas atuais nesse contexto: regulação de custos, maximização da eficiência, alocação de recursos, auto-regulação dos profissionais, prestação de contas etc. Pretendemos não cair na idéia das instituições enquanto fatos sociais totais (Goffman, 1996), desconsiderando o contexto e a sociedade em que se inserem, os grupos de interesses em conflito, as diferenças de acesso aos serviços, motivadas tanto por barreiras organizacionais ou financeiras, quanto por características da população local.

Na perspectiva de criar as bases metodológicas para tanto, vimos a necessidade da revisão dos principais modelos propostos para descrever os determinantes de saúde, os serviços de saúde ou o sistema de saúde em geral. A compreensão do contexto em que surgem e das categorias analíticas que os compõem, buscando contribuições e reconhecendo limitações, parece-nos fundamental para que consigamos contribuir, no campo da análise comparada, com um novo aporte teórico.

Foge ao escopo deste estudo aprofundar uma discussão de cunho epistemológico dos diferentes referenciais que informam esses modelos. No caso específico das correntes em análise comparada, limitamo-nos a sintetizar elementos de trabal ho anterior sobre o tema, por nós já realizado (Conill et al., 1991). Finalmente, tecemos considerações explicitando problemas e encruzilhadas com os quais deparamos.

\section{Considerações metodológicas} para a construção do modelo

A trajetória dos determinantes de saúde nos modelos construídos

Sabemos que a procura de uma resposta diante da dor e da incapacidade se confunde com a própria origem do homem (Scliar, 1987). Os meios empregados variaram em cada época, 
conforme o estágio do conhecimento acerca da doença, o grau da divisão do trabalho e da delegação maior ou menor dessa tarefa a especialistas, e, no que diz respeito ao acesso a um conjunto de bens e serviços disponíveis, as relações estabelecidas entre as classes sociais.

A importância que assumiu a atenção médica nos sistemas de saúde de alguns países desenvolvidos é particularmente bem descrita por Schieber \& Poullier (1989, apud Evans et al., 1996:37):

"As pessoas cuidam de sua saúde, e elas têm razão quando o fazem. De várias formas elas procuram manter, mel horar ou ainda adaptarem-se à sua deteri oração (...) el as parti ci pam de todo tipo de atividade que supostamente contribua para sua boa saúde. Tentam também evitar atividades esituações quejulguem potencial mente perigosas. O que motiva todos esses efforços são teorias ou, mais precisamente, conjuntos de hi póteses implícitas, vagamente relacionadas entresi deforma pouco coerente, sobre os determinantes da saúde.

Dentre as ações que têm por obj etivo a saúde, as sociedades contemporâneas destinam uma grande parte de seus recursos econômi cos à produção edistri bui ção de cuidados médi cos, ou seja, um conjunto de bens eserviços percebidos como relacionados especificamente com a saúde. A atividadedecuidados (...) constitui um dos mais importantes setores da ati vidade econômica em todos os Estados Modernos. A amplitude dos esforços reflete bem a convicção geral de que a disponibilidade de serviços de saúde esua utilização constituem um fator essencial para a saúde de indivíduos bem como de populações."

A compreensão da inter-relação entre os serviços de saúde e o estado de saúde da população precede a discussão de uma nova modelagem para a análise comparada. A descrição dos principais modelos existentes permitirá entender melhor tanto a evolução histórica do conceito de saúde e doença, quanto a forma como os países responderam às necessidades de saúde no plano de organização de seus serviços.

Nesse sentido, escolhemos o modelo ecológico da doença, que deu origem ao modelo epidemiológico clássico da história natural da doença (Leavell \& Clark, 1965), o modelo de multicausalidade ou de determinantes de saúde (Lalonde, 1974), o modelo de sistema de saúde desenvolvido por Roemer (1989) e o que vamos denominar de modelo ampliado e interativo entre circuito de estados de saúde e subsistema de atenção à saúde (Contandriopoulos, 1990).
De acordo com o modelo ecológico da doença, estar doente ou saudável depende do equilíbrio entre algumas variáveis agrupadas em três categorias fundamentais: o agente (organismos infecciosos, agentes físicos, agentes químicos); o hospedeiro (excessos e deficiências dietéticas, idade, sexo etc.) e o meio ambiente (temperatura, umidade, água, alimentos, insetos, vetores, condições econômicas e sócio-culturais). Em razão das interações entre essas numerosas variáveis, o rompimento do equilíbrio em favor de um agente causal levaria o indivíduo ou a população à condição de doente.

Predomina, nessa época, a ênfase no agente para explicar a doença e as doenças infecciosas ainda são a principal preocupação dos epidemiologistas. As propostas de intervenção e organização dos serviços se baseiam em campanhas de erradicação, como, por exemplo, da varíola.

Samuel Pessoa (Pessoa, 1983) caracteriza uma exceção. Num texto intitulado Ecologia e Epidemias, originário de uma conferência realizada na Faculdade de Medicina de Alagoas em 1956 e publicado em 1983, ele argumenta que o desaparecimento ou a diminuição de grandes epidemias e doenças que assolaram a humanidade não foram decorrentes de intervenções médicas, mas de fatores ditos inespecíficos ligados a mudanças na habitação e alimentação. Demonstra, por meio de dados baseados na história dessas doenças, o que McKeown (1981) viria a quantificar mais tarde, analisando séries de mortalidade de mais de três séculos na Inglaterra.

A ampliação do modelo clássico, aumentando a importância dos fatores ambientais e reduzindo a importância dos agentes infecciosos, ocorre com a mudança dos padrões de doença no enfoque dos estudos epidemiológicos. Essa ampliação altera muito lentamente as concepções de saúde/doença nos meios médicos e no planejamento e organização dos serviços, que permanecerão quase até a década de 70, senão até hoje, influenciados pela hipótese da "causa única/efeito único" (Dever, 1988:2-3).

O documento intitulado A New Perspective on the Health of Canadians (Lalonde, 1974), que servirá de base para as ações governamentais no Canadá a partir dessa data, torna-se um marco da perspectiva multicausal no campo das políticas de saúde. Nele, a saúde é considerada como sendo determinada por um conjunto de fatores agrupados em quatro grandes categorias: estilo de vida, ambiente, organização dos cuidados e biologia humana. 
O estilo de vida ou, mais exatamente, os riscos criados pelas próprias pessoas comportam as atividades de lazer, os padrões de consumo e as atividades ocupacionais e de participação na produção; envolvem o conjunto de decisões tomadas pelos indivíduos as quais afetam sua própria saúde. O ambiente é definido como eventos externos ao corpo, compreendendo as dimensões física, social e psicológica. Na biologia humana, influem, além da herança genética, situações tais como a maturidade e o envelhecimento. Por último, o sistema de organização dos serviços é dividido em serviços preventivos, curativos e de recuperação.

Uma das principais fontes conceituais desse documento foi o trabal ho de Laframboise (1973), no qual esse autor propõe uma nova estrutura conceitual para a análise do campo da saúde, com grandes subdivisões passíveis de orientar uma intervenção concreta.

Blum (1974, apud Dever, 1988) dá continuidade à discussão com um modelo denominado "o campo de força e os paradi gmas de bem-estar da saúde", onde confere importância diferenciada a esses quatro grupos de fatores. Tais fatores se relacionam entre si, influenciandose mutuamente num círculo abrangente que inclui população, sistemas culturais e recursos. Por esse modelo, os serviços de saúde têm uma influência menor do que os demais fatores.

Partindo do modelo proposto por Laframboise e Lalonde, Dever propõe, em 1976, o chamado "modelo epidemiológico para análise da política desaúde", no qual os quatro grupos de fatores têm o mesmo peso, precisando estar hipoteticamente em equilíbrio, para que se estabeleça a condição de saúde. A criação desse modelo foi intencionalmente dirigida para que ele fosse um instrumento de orientação à formulação de políticas e ao estabelecimento de prioridades nas ações.

Sua aplicação prática subentende quatro etapas principais: seleção de doenças que contribuam para a mortalidade e morbidade da população; distribuição dos fatores de risco da doença pelas quatro divisões do modelo epidemiológico; distribuição proporcional dos gastos totais com a saúde pelas quatro divisões do modelo de determinação, com posterior readequação da diferença proporcional entre os determinantes da saúde e os gastos implementados (Dever, 1988).

O conceito de campo de saúde que Dever aprimora é mais operacional e tenta se adequar às políticas e à visão de causa múltipla efeito múltiplo de saúde e doença. Seu maior marco éa investigação por igual do estilo de vi$\mathrm{da}$, elementos ambientais e biológicos, assim como de fatores relacionados à organização dos serviços de saúde.

Finalmente, consideramos útil citar o modelo desenvolvido por Roemer (1989), que é uma das mais sintéticas representações existentes de sistema de saúde. De acordo com esse modelo, para responder às necessidades de saúde, o sistema se constitui de três componentes centrais: recursos, organização e prestação de serviços. Esses componentes são influenciados por dois outros fundamentais: a gestão e o financiamento, surgindo dessa interação os resultados do sistema. O autor situa os serviços de saúde como sendo um dos dois grandes determinantes do estado de saúde da população, no mesmo nível de importância do estatuto biológico, este, por sua vez, sendo determinado pelo ambiente físico e social.

Em síntese, notam-se mudanças consideráveis ao longo das últimas décadas no enfoque proposto para identificar os determinantes de saúde. Observa-se a ênfase no agente para explicar a doença, passando pela ampliação desse enfoque, que inclui o meio ambiente num primeiro momento e, posteriormente, um conjunto de fatores (estilo de vida, ambiente, organização dos cuidados e biologia humana). A aplicação desses modelos para a organização dos serviços de saúde passa pela discussão dos limites dos serviços de saúde na melhoria da saúde de uma população, que veremos a seguir.

\section{Os limites dos serviços de saúde}

Uma grande questão na construção de um modelo para análise comparada que se pode antever pelas posições aparentemente antagônicas de Blum (Blum, 1974, apud Dever, 1988), Dever (1988), Lalonde (1974), Laframboise (1973) e Roemer (1989) é exatamente a do peso dos serviços de saúde na melhoria da saúde de uma população.

Embora seguidamente discutida, a questão da importância dos serviços de saúde na produção da saúde tem sido pouco investigada pela sua evidente complexidade. Os autores que se dispuseram a fazê-lo tratam de selecionar variáveis setoriais e extra-setoriais que influenciam o estado de saúde, aplicando então um modelo de regressão logística, de análise multivariada, para identificar aquelas de maior correlação com os melhores resultados.

Um exemplo clássico é o estudo de Cochrane et al. (1988), no qual selecionaram-se 18 países, excluindo-se o Japão para evitar a interferência de fatores genéticos na explicação das diferenças observadas na mortalidade. Os cri- 
térios de seleção foram: produto nacional bruto por pessoa superior a dois mil dólares; população superior a dois milhões de habitantes; disponibilidade de dados. Analisaram-se as relações entre indicadores de atenção à saúde (recursos humanos/ habitantes, percentual do PNB gasto em saúde, leitos/ habitantes) e dietéticos (consumo alimentar, de fumo e de ál$\mathrm{cool} /$ anual/per capita), bem como fatores econômicos e demográficos (densidade populacional, PNB/per capita, índice de educação), com as diversas taxas de mortalidade de 0-64 anos.

As conclusões foram polêmicas. Os indicadores de atenção à saúde não se relacionavam negativamente com a mortalidade, existindo uma associação marcada e positiva entre a prevalência de médicos e a mortalidade no grupo de idades mais jovens. Não se encontrou nenhuma explicação para essa "anomalia de médicos". O produto nacional bruto por habitante é a principal variável que mostra uma associação negativa constante e forte com a mortalidade.

Uma contribuição interessante nesse sentido é a hipótese que Miguel (1978), utilizandose de métodos quantitativos, apresenta para a Espanha no período entre 1960 e 1970 . O autor argumenta que, em formações sociais com baixo nível de desenvolvimento, as diferenças no estado de saúde podem ser melhor explicadas pelo grau de mudança econômica do que pela estrutura e organização de serviços. Em estágios mais avançados de desenvolvimento, os serviços passam a constituir-se numa variável independente, de maior importância para o nível de saúde alcançado do que o crescimento econômico.

A fim de superar essa problemática, Contandriopoulos (1990) propõe um modelo ampliado, onde interagem dois circuitos: o que determina o estado de saúde e o que determina o que ele chama de sistema de cuidados (Figura 1). São cinco os principais determinantes do estado de saúde da população: a biologia humana, os hábitos de vida, o desenvolvimento físico e social e os serviços de saúde. Além dessa vantagem, o modelo possibilita estabelecer relações entre os diversos componentes, permitindo medidas de avaliação do sistema de serviços, tais como produtividade, efetividade e eficiência.

Entendendo a saúde como um estado dinâmico, os serviços devem ter como função principal alterar o processo natural de evolução da doença. A medida e a definição exatas do que é um problema de saúde estão relativizadas, tanto pelo agente que a percebe (o usuário, a sociedade, o Estado, os prestadores do sistema de saúde), quanto pela própria eficácia dos serviços. Quanto mais o sistema se aprimora, mais se amplia a definição do que se constitui um problema de saúde, justificando seus desenvolvimento e aumento de custo.

Por esse modelo, os serviços seriam responsáveis pela gerência eficiente dos recursos empregados pela sociedade. Isso se faria de acordo com regras e valores organizacionais aceitos de forma a atender às expectativas e contribuir o máximo possível para a melhoria da saúde da população.

O modelo proposto inclui uma concepção influenciada pelo modelo de Lalonde (1974), mas ampliada, dos determinantes da saúde de uma população e do papel real do chamado subsistema de atenção à saúde sobre ela. As diferentes modalidades organizacionais nos diferentes países (responsabilidade dos cidadãos, do Estado, regulamentação profissional, tipos de financiamento e pagamento pelos serviços) podem acarretar as principais diferenças entre seus sistemas de saúde, na medida em que influenciam os papéis dos diferentes atores sociais e repercutem na capacidade de gerir os gastos e na eficiência dos serviços para a população.

Aliás, é importante atentar para o fato de que a lógica que rege as particularidades funcionais do sistema de saúde pode não necessariamente estar voltada para o bem-estar da população, visto que o comportamento dos diversos (e por vezes antagônicos) atores sociais dentro de tal sistema pode direcionar sua dinâmica para outros fins.

Usando esse referencial, Champagne et al. (1991) comparam a evolução dos serviços de saúde do Canadá e dos Estados Unidos com a dos outros países da Organização para a Cooperação Econômica e o Desenvolvimento (Oced), analisando a produtividade, a efetividade e a eficiência desses sistemas. Como indicadores de recursos, usam o percentual do PIB em saúde e despesas per capita; para os serviços, o tempo de permanência hospitalar e o número de médicos por habitantee, para os resultados, a esperança de vida e a mortalidade infantil. Demonstram as dificuldades do sistema americano, com gastos mais elevados e piores indicadores de saúde, caracterizando-o como o menos eficiente de todos.

A discussão dos diferentes determinantes do estado de saúde é fundamental, na medida em que traz a questão de como separar e medir os efeitos dos componentes de um sistema de saúde (dentre os quais os serviços), não somente no que se refere à análise comparada, mas em relação a toda a discussão da organi- 
Figura 1

Modelo de sistema de saúde.

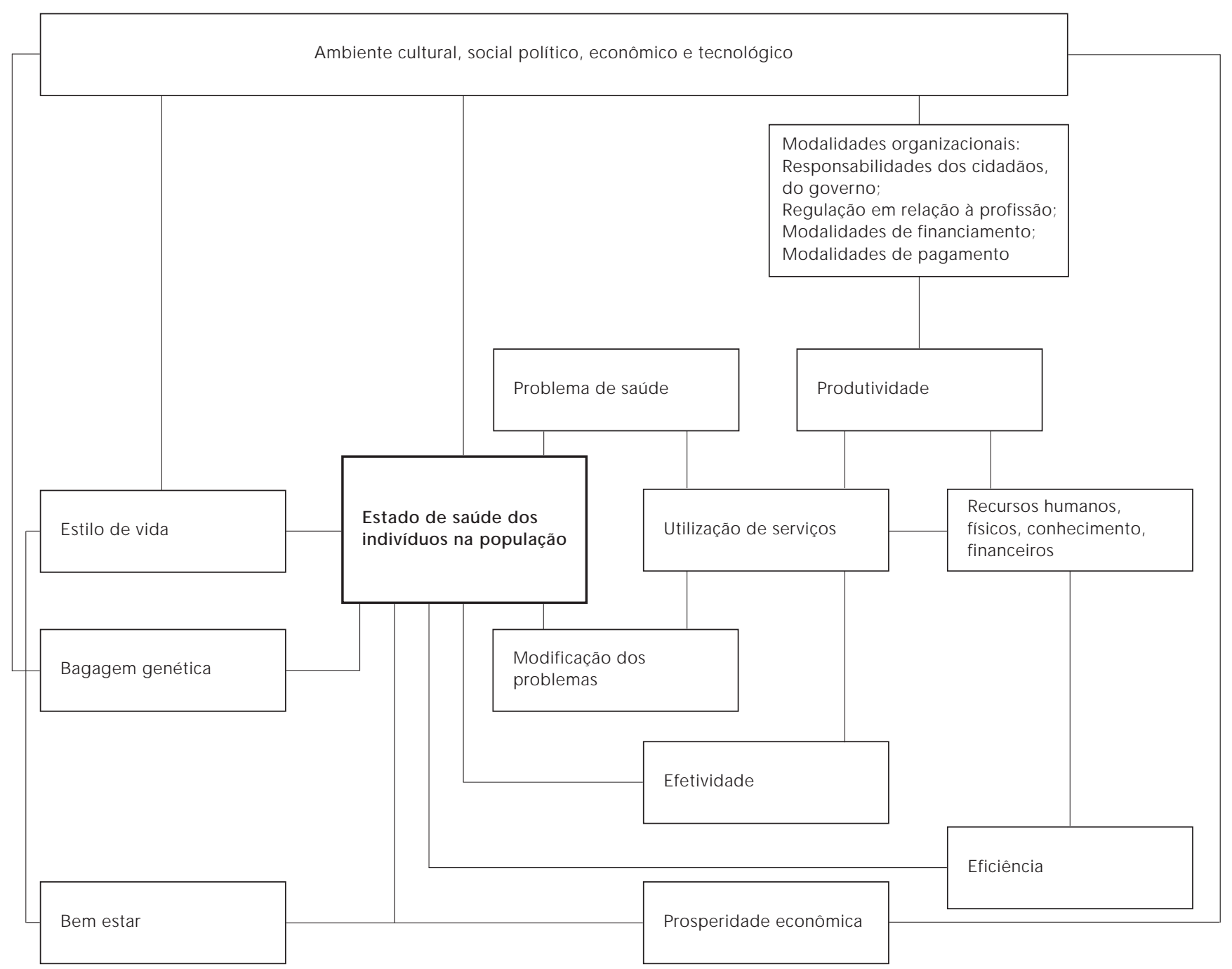

Fonte: Contandriopoulos, A. P., 1990 (com autorização).

zação dos serviços influenciada por atividades extra-setoriais (educação, renda, acessibilidade geográfica etc.).

\section{A bordagem comparada em saúde e os cuidados na construção do modelo}

Entendemos a comparação como a busca de semelhanças, diferenças ou relações entre fenômenos que podem ser contemporâneos ou não, que ocorram em espaços distintos ou não, tendo em vista conhecer determinações, causalidades e inter-relações (Conill et al., 1991).
A abordagem comparada é um instrumento metodológico importante; no entanto, tem utilizado enfoques de descrição e análise sob bases filosóficas diferentes. Recente revisão dos enfoques utilizados na área da saúde (Conill et al., 1991) dividiu-os em três correntes: funcionalismo, materialismo-histórico e "novas correntes". Na primeira, predominam a descrição, a classificação e o estabelecimento de indicadores para explicar a estrutura e o funcionamento dos diversos sistemas oficiais de atenção à saúde. Destacam-se os trabalhos de Field (1973), Frenk \& Donabedian (1987), Klecskowski et al. (1984) e Roemer (1989). 
A segunda caracteriza-se por uma quase ausência de esquemas e tipologias e por estudos que basicamente procuram ver as relações do imperialismo com a assistência à saúde, a organização dos serviços nos países em transição para o socialismo e as contradições das reformas capitalistas. Os trabalhos mais destacados são os de Navarro (1976, 1977, 1983).

A terceira, descrita como "novas correntes" (Illich, 1975; Capra, 1982; Gorz, 1987), utilizase da comparação internacional para analisar os efeitos da industrialização sobre os sistemas de saúde, que tenderiam a uma convergência da problemática, independentemente da formação social na qual se situam. Na tentativa de impedir os efeitos da iatrogênese estrutural (IIlich, 1975), que retira gradativamente dos indivíduos a capacidade de criar seus próprios valores de uso, deve-se diminuir a profissionalização, a burocratização e conseqüentemente, frear a expansão desses sistemas.

Mesmo levando em consideração a variação metodológica entre os enfoques, é possível dizer que os planos em que estas análises foram realizadas e a sistematização das categorias utilizadas pouco se voltaram para o conhecimento dos resultados atingidos na implantação e implementação dos sistemas de saúde.

Acreditamos que as dificuldades podem ser superadas com a incorporação dos vários elementos das diversas correntes no processo sintético (e dialético) de incorporação de divergências. Ao desenvolvermos uma metodologia que supere a tendência fragmentadora do funcionalismo e a ortodoxia do materialismo-histórico e ao redefinirmos categorias e indicadores a serem comparados, estaremos buscando a superação das dualidades, polarizações e dicotomias que tradicionalmente têm marcado a produção do conhecimento na área (Conill et al., 1991).

Considerando os objetivos deste trabalho, adota-se o seguinte conceito de sistema de saúde: "conjunto de relações entre insti tuições, grupos sociais e indivíduos, orientados para a manutenção e mel hora do nível de saúde de uma população humana determinada" (Miguel, 1978:142). Quanto ao serviço de saúde, partese do pressuposto de que ele seja o resultado de produções anteriores, acumulação de conhecimentos, representações, procedimentos, técnicas e riquezas, um conjunto sedimentado de forma mais ou menos estável, onde somente uma minoria é suscetível a adaptações a curto prazo (Eraly, 1988).

Uma vantagem potencial da análise comparada é a possibilidade de incluir variáveis históricas, político-administrativas e culturais em suas especificidades, o que permite ampliar o leque de relações de determinação e observar as respostas dadas em determinado contexto. Além disso, uma análise comparada que leve em conta algumas categorias básicas e um grande número de indicadores tem a vantagem de permitir a construção de tipologias e modelos que serão confrontados, por seu lado, com outras pesquisas empíricas para confirmação, ajustes ou até mesmo seu abandono.

Mas as desvantagens precisam ser também apontadas. Uma delas é a ausência de dados homogêneos de um país a outro para análises de perfil quantitativo (sistema de informações diferentes). Outra desvantagem é a escolha forçosa entre uma grande quantidade de indicadores a serem utilizados num pequeno recorte espacial ou temporal, o que pode restringir a capacidade de comparação ou, pelo contrário, optar-se pela extensão do estudo, levando a perdas no refinamento e aprofundamento dos resultados.

Em síntese, no desenvolvimento do modelo que pretendemos, é preciso levar em conta pelo menos dois princípios: deve ser aplicado a formações sociais com indicadores sócio-econômicos relativamente semelhantes e deve-se ter a clareza de que permanecemos em nível do subsistema de cuidados.

Um modelo é uma estrutura analítica que estuda as relações entre um conjunto de variáveis, sendo composto de uma ou mais hipóteses com base nas quais retiramos implicações ou previsões. Preconiza-se que, para ser útil, o modelo deve ser uma versão simplificada e abstrata da realidade (Netto, 1986). Demo (1982), numa análise crítica da construção de modelos como método, proposto por Lévi-Strauss, afirma que o modelo seria sempre simplificador ao tentar "cristalizar em um núcleo concatenado e estável a superfície complexa da realidade, apoiando-se na crença de que a subjacência, sendo ordenada (estruturada) esi mples, contém a explicação do fenômeno" (Demo, 1982:213). Se o modelo for tão complexo quanto a própria realidade, ele perde sua força explicativa, força esta que seria obtida na decomposição analítica das invariantes subjacentes. Neste caso, segundo Demo, o desafio é de que "o mais si mples deve explicar mais, desde que dê conta de toda a realidade" (Demo, 1982:213).

Tendo definido então o que é um modelo e sendo o nosso objeto de estudo a organização dos serviços de saúde, passamos a questão seguinte: como construí-lo?

Partindo do pressuposto de que os serviços de saúde são fenômenos complexos, por causa tanto de seus elementos estruturantes, quanto 
das ações e interações inter e intra-organizacionais, consideramos o modelo processual (sistêmico) proposto por Le Moigne (1990) o mais apropriado.

De acordo com este autor, modelar um sistema complexo é modelar um sistema de ação. A modelagem sistêmica parte da seguinte questão: O que é feito? Quais são as funções e transformações ou as operações asseguradas ou a assegurar? Utilizar esse referencial implica encarar uma dinâmica de ação que se desenrola no tempo, que desloca seus produtos e a si mesma num espaço e que afeta a forma desses produtos. Exige toda uma contextualização dentro de um referencial tempo-espaço-forma específico.

Utilizaremos a modelagem sistêmica mais simples, que é a que estabelece a relação entre os recursos, os serviços produzidos e os resultados (Donabedian, 1972).

Nossas perguntas devem questionar como os serviços são produzidos. As categorias escoIhidas, em vez de descreverem componentes, devem ser vistas como características do processo de produção de serviços.

Dever (1989:10) cita as seguintes vantagens da utilização do modelo por ele concebido, que devem ser levadas em conta na construção de um modelo:

- possibilidade de se dar igual destaque a categorias localizadas em diferentes dimensões; - sua abrangência, isto é, a possibilidade de se fazer a descrição ou a análise, valendo-se de cada categoria do modelo ou da combinação delas;

- possibilidade de identificar padrões, examinando a importância relativa de cada uma das categorias;

- possibilidade de explorar áreas anteriormente negligenciadas.

O cerne da questão localiza-se, então, na escolha e na operacionalização das categorias de interesse e na seleção dos indicadores a serem utilizados.

N otas preliminares sobre as categorias e indicadores para a operacionalização do modelo

Em relação à escolha das categorias, surgem as seguintes questões. Em primeiro lugar, que dêem conta de uma abordagem global do modelo escolhido e lhe confiram coerência, o que pressupõe base conceitual precisa e limites adequados aos âmbitos de cada questão. Em segundo, que considerem um recorte espacial e temporal para a aplicação do modelo (um país, estados, municípios).
Os critérios utilizados para a escolha de indicadores de monitoramento do sistema de saúde canadense (Chevalier et al., 1995) podem ser lembrados:

- devem ser úteis à tomada de decisões;

- devem ser sensíveis, isto é, seus valores devem variar em função do objeto que eles querem medir;

- devem permitir comparações temporais;

- devem apresentar uma certa variabilidade no tempo e no espaço;

- devem ter um caráter de exclusividade mútua, isto é, não devem se superpor entre si;

- devem ter boa reputação, o que Ihes confere validade a priori.

As categorias descentralização e acesso são categorias universalmente presentes nas análises de sistemas de saúde e aparecem como promissoras na construção do modelo.

A categoria descentralização vem a ser um dos principais componentes das transformações que vêm ocorrendo nos princípios constitucionais e nas estruturas financeiras e organizacionais de alguns países. Para Contandriopoulos (1994), a descentralização é uma das idéias mestras de uma terceira via na saída da crise pela qual o setor passa.

Entendemos que a descentralização pode criar as bases para uma maior autonomia local no uso dos recursos e na implementação de políticas. De uma maneira geral, uma modalidade de organização de serviços que se caracterize por ser descentralizada deve comportar um maior controle do nível local no processo de decisão, mesmo se tratado como um continuum ou condicionado por seu maior ou menor grau de descentralização (Hortale, 1997).

No plano da administração pública, um serviço descentralizado pode se caracterizar por transferir tanto poderes de decisão, quanto de autoridade, qualquer que seja a dimensão. Na dimensão territorial, pode caracterizar-se pela maior proximidade com o nível local dos serviços, onde haja maior acesso e controle por parte dos setores organizados, e na dimensão funcional, pela possibilidade de criar espaços de maior autonomia e participação.

Em síntese, um serviço descentralizado possibilita o equilíbrio entre responsabilidade, obrigações e poderes do nível central e local. Do ponto de vista do modelo, pode ser verificado mediante a alocação dos recursos para a produção dos serviços. A hipótese a ser verificada é se a descentralização relaciona-se com uma melhor adequação do acesso aos serviços.

A definição do conceito de acesso tem sido problemática, pois existe uma infinidade de sinônimos para o termo: disponibilidade de re- 
cursos financeiros e de saúde numa zona determinada; serviços disponíveis no momento e lugar em que o paciente necessita, onde a forma de entrada no sistema seja clara; acessibilidade financeira (capacidade individual para custear a atenção médica); acessibilidade física (custos de transporte, tempo e busca), grau de ajuste entre as características da população e dos recursos de atenção à saúde, dentre outros (Aday \& Andersen, 1992).

Para esses autores, o termo acesso tem representado mais um conceito político do que operativo (ter acesso ou entrar no sistema constitui, explícita ou implicitamente, um objetivo das políticas de saúde), tendo sido feitos "poucos esforços para elaborar uma defini ção sistemática conceitual ou empírica, que pudesse possibilitar aos responsáveis pela tomada de deci sões e aos consumi dores aval iarem a efetividade dos diferentes programas para al cançar essa meta" (Aday \& Andersen, 1992).

A categoria acesso deve, portanto, ser vista não somente como geográfica e econômica, mas também organizacional e sócio-cultural. Ela pode descrever a capacidade que um um serviço tem de dar cobertura a uma determinada população, ou o obstáculo a sua utilização, caracterizando uma interação que ocorre em um processo de produção de serviços.

Para Aday \& Andersen (1992), foi Donabedian quem melhor definiu a categoria acesso: "a prova de acesso consistena utilização de um serviço, não si mplesmente na sua existência". Dessa forma, “o acesso pode ser medido pelo nível de utilização em relação à necessidade. Devemos reconhecer que a aval i ação da necessi dade dos clientes difere da dos profissionais. Além disso, é preciso distinguir dois componentes na utilização do serviço: o início e a continuidade" (Donabedian, apud Aday \& Andersen, 1992).

Analisar o acesso em termos de custo, disponibilidade, economia interna (tempo de espera, demora e interrupções na prestação de serviços etc.) e variáveis psicológicas ou conhecimento sobre a saúde não é suficiente para indicar se todos aqueles indivíduos que desejam entrar no sistema efetivamente conseguem. Embora seja importante conhecer em que grau esses fatores determinam uma diferença na obtenção da atenção, a principal dificuldade ao avaliar o acesso é verificar se as pessoas que realmente necessitam de atenção receberam-na. Esse aspecto permanece, em geral, não resolvido na bibliografia consultada (Aday \& Andersen, 1974; Donabedian, 1972).

\section{Considerações finais}

Ao longo do artigo, identificamos alguns entraves metodológicos que devem ser superados e que resumimos a seguir.

Um deles é a adequação do modelo à realidade social versus a sua simplificação. Um dos desafios é exatamente este: nem incorrer no ilustracionismo racionalizador que percorre só o caminho positivista de comprovação na prática das hipóteses teóricas (teoria $\rightarrow$ prática), nem acharmos que os dados falarão por si (prática $\rightarrow$ teoria). As questões teóricas emergem dos desafios da realidade e a eles retornam para uma confrontação, remodelagem, aperfeiçoamento e utilização (prática $\rightarrow$ teoria $\rightarrow$ prática) (Freire, 1970).

O outro entrave refere-se à heterogeneidade na operacionalização das políticas públicas nas diferentes formações sociais. Como estabelecer comparações que sejam suficientemente sensíveis a essas influências, sem deixarem de ser operativas? Como criar um modelo que consiga separar o setorial, sem restringi-lo em demasia, de forma que se possa continuar tendo alguns critérios operativos capazes de comparar sistemas diversos entre si no plano das políticas públicas? A nossa proposta éa de, num primeiro momento, permanecermos em nível dos resultados do processo de produção de serviço ou em nível da comparação do chamado subsistema de atenção à saúde.

Será a partir de um aprofundamento teórico e de uma primeira sistematização das categorias acesso e descentralização que partiremos para a operacionalização dos seus componentes mais pertinentes na construção do novo modelo, que tem como preocupação tanto a dinâmica dos serviços de saúde, quanto a dimensão avaliativa.

O que nos parece importante ressaltar é que, antes de efetuarmos uma modelagem somente da organização dos serviços, devemos pensar de que forma ela se relaciona com os outros determinantes de um sistema de saúde concebido já de forma ampliada, senão corremos o risco de sub ou superavaliarmos a influência de determinada variável organizacional tomada isoladamente de seu contexto.

Em síntese, essas são algumas questões que mostram o desafio na construção de um modelo para análise comparada da organização de serviços de saúde. 


\section{Agradecimentos}

Ao Prof. François Champagne, da Universidade de Montréal, Canadá, pelo aporte metodológico relevante na discussão dos principais elementos para a construção do modelo.

\section{Referências}

ADAY, L. A. \& ANDERSEN, R., 1992. Marco teórico para el estudio del acceso a la atención médica. In: Investigaciones sobre Servicios de Salud: Una Antología (K. L., White, ed.), pp. 604-613, Washington: OPAS.

CAPRA, F., 1982. O Ponto de Mutação. São Paulo: Cultrix.

CHAMPAGNE, F.; CONTANDRIOPOULOS, A. P.; LEM AY, A. \& PREKER, A., 1990. The evolution of health care performance in Canada, the U.S. and other OECD countries: a comparative analysis of productivity, effectiveness and efficiency. Groupe de Recherche Interdisciplinaireen Santé, 18:1-17.

CHEVALIER, S.; CHOINIĖRE, R.; FERLAND, M.; PAGEAU, M. \& SAUVAGEAU, Y., 1995. Indicateurs Sociosanitaires: Définitions et Interprétations. Ottawa: Institut Canadien d'Information sur la Santé.

COCHRANE, A. L.; LÉGER, A. S. S. \& MOORE, F., 1988. Entradas de atención de salud y salidas de mortalidad en los países desarrolados. In: El Desafío de la Epidemi ol ogía, Problemas y Lecturas Seleccionadas (C. Buck, A. Lopes, E. Nájera \& M. Terris, orgs.), pp. 1.015-1.022, Washington: Organización Panamericana de la Salud/Organización Mundial de la Salud.

CONILL, E.; MENDONÇA, M. H.; SILVA, R. R. \& GAWRYSZEWSKI, V., 1991. A organização dos serviços de saúde: a comparação como contribuição. Cadernos de Saúde Pública, 7:328-346.

CONTANDRIOPOULOS, A. P., 1990. Recherche sur les fondements d'un modèle théorique du système de santé. Groupe de Recherche Interdisci plinaire en Santé, 13:1-11.

CONTANDRIOPOULOS, A. P., 1994. Réformer le système de santé: une utopie pour sortir d'un statu quo impossible. Ruptures, Revue Transdisciplinaireen Santé, 1:8-26.

DEVER, A., 1988. Epidemiologia: enfocando a prevenção. In: A Epidemiologia na Administração dos Serviços de Saúde, pp. 1-24, São Paulo: Prohasa/ Livraria Pioneira e Editora.

ERALY, A., 1988. La Structuration de l'Entreprise. Bruxelles: Editions de l'Université de Bruxelles.

EVANS, R. G. \& STODDART, G. L., 1996. Produire de la santé, consommer des soins. In: Être ou ne pas Êtreen BonneSanté: Biologie et Déterminants Sociaux de la Maladie, pp. 37-73, Paris/Montréal: Presse de l'Université de Montréal/John Libbey Eurotext.

FIELD, M., 1973. The concept of the health system at the macrosociological level. Social Science and Medicine, 7:763-785.

FREIRE, P., 1970. Pedagogia do Oprimido. Rio de Janeiro: Paz eTerra.
FRENK, J. \& DONABEDIAN, A., 1987. State intervention in medical care: types, trends and variables. Health Policy and Planning, 2:17-31.

GOFFM AN, E., 1996. Prisões, Manicômios e Conventos. Rio de Janeiro: Perspectiva.

GORZ, A., 1987. Adeus ao Proletariado - para Além do Socialismo. Rio de Janeiro: Forense Universitária.

HORTALE, V., 1997. O conceito de descentralização aplicado aos serviços de saúde: dimensões, padrões e regularidades. Revista de Administração Pública, 31:23-35.

ILLICH, I., 1975. A Expropriação da Saúde- Nêmesis da Medicina. Rio de Janeiro: Nova Fronteira.

KLECSKOWSKI, B.; ROEMER, M. \& VAN DER WERFF, A., 1984. Si stemas Nacionales de Salud y su Reorientación Hacia Ia Salud para Todos. Serie Cuadernos de Salud Pública, 77. Ginebra: Organización Mundial de la Salud.

LAFRAMBOISE, H. L., 1973. Health policy: breaking the problem down into more manageable segments. Canadian Medical Association Journal, 108:388-393.

LEAVELL, H. R. \& CLARK, G. R., 1965. Preventive Medicine for the Doctor in his Community. An Epidemiologic Approach. 3rd ed., New York: McGrawHill.

LE MOIGNE, J. L., 1990. La Modélisation des Systèmes Complexes. Paris: Dunod.

McKEOWN, T., 1981. Les déterminants de l'état de santé des populations depuis trois siècles: le comportement, l'environement e la médecine. In: Médecine et Soci etéles Annès 80 (L. Bozzini, M. Renaud, D. Gaucher, J. Llambias-Wolff, eds.), pp. 143-233, Montréal: Coop.Saint Martin.

MIGUEL, J. M., 1978. El sistema sanitario. In: Sociología de la Medicina (J. M. Miguel, org.), pp. 139177, Barcelona: Vicens.

NAVARRO, V., 1976. Medicine under Capitalism. New York: Prodest.

NAVARRO, V., 1977. Health and Medical Care in the U.S.: A Critical Analyisis. New York: Baywood Publishing Company Inc.

NAVARRO, V., 1983. Health and Work under Capitalism; An International Perspective. New York: Baywood Publishing Company Inc.

NETTO, A. G. M., 1986. Dicionário de Ciências Sociais. Rio de Janeiro: Fundação Getúlio Vargas.

PESSOA, S. B., 1983. Ecologia e epidemias. In: Ensaios Médico-Sociais (S. Pessoa, org.), pp. 157-175, Rio de Janeiro: Guanabara-Koogan.

ROEMER, M., 1989. National health systems as market intervention. Journal of Public Health Policy, 62-77.

SCLIAR, M., 1987. Do Mágico ao Social. Porto Alegre: LPM. 